\title{
Intérêt et limites de l'épidémiologie pour l'évaluation des risques de cancers radioinduits et l'établissement des normes de radioprotection
}

\author{
D. HUBERT *
}

(Manuscrit reçu le 20 juin 1989)

RÉSUMÉ Les études épidémiologiques concernant des personnes exposées aux rayonnements ionisants restent à la base des estimations de risque de cancer radioinduit chez l'homme. La principale étude ayant servi à l'élaboration des normes de radioprotection est celle des survivants d'Hiroshima et Nagasaki. Les malades traités par radiothérapie ou ayant subi des radiographies dans un but diagnostique, ainsi que des groupes de travailleurs exposés professionnellement fournissent également nombre d'informations sur le risque de radiocarcinogenèse.

Ces études épidémiologiques permettent d'affirmer avec certitude l'existence d'un risque pour certains cancers après irradiation à fortes doses, souvent délivrées à fort débit de dose. Par contre, aucune conclusion n'est possible pour les faibles doses et faibles débits de dose. On est donc obligé d'extrapoler aux faibles doses et taibles débits les risques connus pour les fortes doses en utilisant divers modèles mathématiques pour la relation entre la dose et l'effet observé. Une autre difficulté vient de la grande période de latence des cancers radio-induits, ce qui explique que tous les cancers en excès n'aient pas encore été observés dans les populations irradiées étudiées. Là encore, on a recours à des modèles mathématiques pour projeter sur la vie entière le risque de cancers excédentaires. Les estimations de risque de radiocancers sont, par conséquent, entachées de nombreuses incertitudes, puisqu'elles varient en fonction du modèle utilisé. D'autres incertitudes proviennent des données de base, en particulier de la dosimétrie, et se majorent lorsqu'il s'agit de transposer les risques d'une population à une autre.

L'UNSCEAR, en 1988, a proposé de nouvelles estimations pour le risque de décés excédentaires par cancers sur la vie entière qui serait compris entre 4 et $11 \%$ par gray ; ces valeurs représentent une réévaluation des précédentes estimations d'un facteur 1,6 à 4,4 , et sont en grande partie la conséquence de l'utilisation de modèles de projection différents. D'autre part, elles s'appuient uniquement sur les observations des survivants d'Hiroshima et Nagasaki, alors que les études de malades fournissent un risque moindre. Enfin, l'UNSCEAR ne définit pas précisément le facteur de réduction utilisable pour passer des fortes doses et forts débits de dose aux faibles doses et faibles débits qui reste compris entre 2 et 10. En raison des nombreuses incertitudes persistantes, il ne semble pas justifiè de modifier actuellement les normes de radioprotection.

\footnotetext{
* Electricité de France, Comité de radioprotection, 3, rue de Messine, 75384 Paris Cedex 08.
} 
ABSTRACT Epidemiological studies in subjects exposed to ionizing radiation are mainly used in order to estimate the risk of radiation induced cancer in humans. Protection standards were assessed from the major study of Hiroshima and Nagasaki survivors. Patients treated with therapeutic radiation or receiving diagnostic examinations and occupational groups have also yielded a great deal of information on radiation carcinogenesis risk.

These epidemiological studies allow to confirm that a risk does exist for some types of cancer following high-dose exposures often at high dose-rates. However, no conclusion can be drawn for low doses and low dose-rates. Therefore we have to extrapolate from known high-dose risks to low doses and low dose-rates by various dose-response patterns. Another difficulty in assessing radiation cancer risks comes from the long latency time, which explains that all excess cancers have not yet been observed in the irradiated population studied. Once more, mathematical mojejls are used to project excess lifetime cancer mortality. The estimations of radiation cancer risks are therefore marked by a great number of uncertainties, since they change accordingly to the model used. Other uncertainties come from the data, especially the dose estimates and are heightened when extrapolating to other populations.

In 1988, UNSCEAR assessed new estimates for excess lifetime cancer mortality in the range of 4 to $11 \%$ per gray. These values mean a revaluation of the previous estimates by a 1.6 to 4.4 factor, which is mainly consecutive to the use of different projection models. Besides, they are solely based on the Hiroshima and Nagasaki survivors, whereas patient studies assess a lower risk. Finally UNSCEAR does not precisely state what is the available reduction factor to modify risks for low doses and low dose rates which should lie between 2 and 10. Due to a number of persistant uncertainties, we should not consider it justified to revise protection standards presently.

\section{INTRODUCTION}

Les rayonnements ionisants peuvent favoriser chez l'homme la survenue de cancers à distance de l'irradiation, même lorsqu'ils n'ont entraîné aucun effet somatique immédiat. Mais leur risque cancérigène reste très difficile à évaluer: d'une part en raison de la période de latence prolongée précédant l'apparition du cancer (au moins 10 ans pour les tumeurs solides), et d'autre part du fait de l'absence de spécificité des cancers radioinduits [32]. II ne s'agit que d'une augmentation de fréquence de certains cancers, déjà observés en l'absence de toute irradiation.

Deux types de données permettent une meilleure approche du risque cancérigène lié aux rayonnements ionisants:

(1) Celles provenant de l'expérimentation animale qui fournit des modèles intéressants, permettant d'élaborer des hypothèses pour les relations dose-effet $[23,28]$; elles restent néanmoins limitées par la difficulté de transposer les faits observés à l'espèce humaine, les effets des rayonnements étant très variables d'une espèce à l'autre;

(2) Les enquêtes épidémiologiques effectuées dans des groupes de populations exposés aux rayonnements ionisants constituent la base principale pour le calcul des facteurs de risque de cancer après irradiation [22].

Or, il est important de pouvoir établir une estimation du risque la plus précise possible, dans la mesure où le calcul des normes de radioprotection se fonde, en grande partie, sur ces évaluations de risque de cancer après irradiation. 
Après un rappel des sources de données disponibles, nous nous intéresserons aux méthodes utilisées pour évaluer les risques cancérigènes liés aux rayonnements ionisants, en analysant les nombreuses incertitudes qui s'y attachent. Nous passerons en revue les différentes valeurs proposées par le Comité scientifique des Nations Unies pour l'étude des effets des rayonnements ionisants (UNSCEAR) en 1988 pour ces risques de cancer, en considérant séparément le problème des faibles doses $[28,44]$. Comparant ces chiffres aux valeurs précédemment proposées par I'UNSCEAR ou d'autres organismes internationaux, nous discuterons les différences observées et les implications pour les normes de radioprotection.

\section{SOURCES DE DONNÉES}

Plusieurs groupes de population ont été exposés aux rayonnements ionisants, soit de façon accidentelle, soit pour une raison médicale, ou encore dans le cadre de leur profession. L'étude de ces populations constitue une des bases pour l'étude des effets à long terme des rayonnements. Une revue détaillée des enquêtes épidémiologiques disponibles a été mise à jour par I'UNSCEAR [44]. Nous en citerons une partie à titre d'exemples.

\section{I.1. Expositions militaires et accidentelles}

\section{I.1.1. Explosions nucléaires à Hiroshima et Nagasaki}

La population japonaise exposée aux bombardements atomiques d'Hiroshima et Nagasaki a fourni une somme considérable de données. En 1950, une cohorte de 120000 survivants a été identifiée dans le cadre de l'enquête sur la longévité (Lide-Span Study ou LSS) et suivie de façon très régulière. En particulier, tous les décès et leurs causes ont été recensés [37]. Des calculs de dose ont été effectués individuellement en 1965 pour 91228 personnes de la cohorte totale (dosimétrie TD 65) et en 1985, 7096 étaient décédées par cancer. Récemment, on s'est rendu compte qu'il y avait eu des erreurs d'évaluation dans cette dosimétrie TD 65. De nouvelles doses individuelles ont par conséquent été calculées dans le cadre de la dosimétrie DS 86 , mais ceci n'a été jusqu'à présent possible que pour 75991 personnes constituant une sous-cohorte de la cohorte LSS TD 65; parmi elles 5936 sont mortes de cancer entre 1950 et 1985 [38].

L'intérêt majeur de cette étude tient à l'importance de la population touchée et à sa diversité, sans qu'il existe de sélection d'âge, de sexe ou de radiosensibilité particulière. En contrepartie, l'inconvénient principal, pour la transposition du risque à d'autres populations, vient du mode d'irradiation: il s'agissait d'une irradiation aiguë brève à très fort débit de dose, ce qui est très différent des conditions d'irradiations intéressant la radioprotection, à savoir les irradiations à faibles doses et faibles débits de dose.

\section{l.1.2. Accidents d'irradiation}

- Les habitants des îles Marshall (250 personnes) et 23 pêcheurs japonais ont été exposés en 1954 aux retombées d'essais d'armes thermonucléaires américaines [17]. 
- Les personnes irradiées en 1986 à la suite de l'accident de la centrale nucléaire de Tchernobyl en URSS font l'objet d'une surveillance rigoureuse mais il faudra attendre de nombreuses années avant de pouvoir tirer des conclusions de cette enquête épidémiologique [21, 45].

- D'autres accidents radiologiques, la plupart dus à la manipulation défectueuse de sources industrielles de gammagraphie ou à une exposition aux rayons $X$ utilisés pour des contrôles de qualité, sont répertoriés dans un registre mondial (1 100 personnes dont 38 décès). Mais ces données sont difficilement utilisables en raison du petit nombre de personnes concernées par chaque accident et des conditions d'irradiation souvent variables d'un accident à l'autre.

\section{I.2. Irradiations médicales}

On distingue:

- les groupes de personnes traitées par radiothérapie, le plus souvent en raison d'un cancer, mais aussi pour des affections bénignes à une période où l'on méconnaissait les risques cancérigènes des rayonnements ionisants;

- les nombreux examens radiologiques à visée diagnostique touchant une très vaste population.

\section{I.2.1. Radiothérapie pour affection maligne}

La radiothérapie contribue au traitement et à la guérison de nombreux cancéreux. Or, il est désormais possible d'évaluer de façon précise la dose reçue par les différents organes, même ceux situés loin de la région irradiée mais atteints par le rayonnement diffusé. Les chances de survie ayant augmenté, on dispose d'un nombre croissant de données sur les risques de second cancer, en particulier chez les individus traités pour cancer de l'enfance.

Une enquête est particulièrement importante car elle regroupe $\mathbf{8 3 0 0 0}$ femmes irradiées pour cancer du col de l'utérus. L'étude initiale de cohorte [4] permettant des estimations de coefficients de risque en fonction des doses de rayonnements reçues a été complétée par des études castémoins pour les leucémies [3] et les autres cancers [5].

\subsubsection{Radiothérapie pour affection bénigne}

Au Royaume Uni, 14000 personnes irradiées pour une spondylarthrite ankylosante ont été très régulièrement suivies et cette population a également permis d'établir des calculs précis de risque de leucémie ou de cancers secondaires $[13,39]$. Une nouvelle estimation des doses absorbées aux différents organes devrait accroître la fiabilité des évaluations de risque pour cette cohorte [25]. 
D'autres populations, particulièrement des enfants, ont été irradiées au niveau de la tête et du cou pour hypertrophie du thymus, pathologie thyroïdienne ou teigne du cuir chevelu. Ailleurs, l'irradiation était indiquée pour hémangiome cutané ou, chez la femme, pour une mastite.

\subsubsection{Radiodiagnostic}

Les enquêtes sur l'effet des examens radiologiques touchent des dizaines de milliers de personnes. Citons l'enquête effectuée chez des femmes ayant subi de nombreuses radioscopies thoraciques pour surveillance de pneumothorax (à l'époque, moyen de traitement de leur tuberculose pulmonaire) et ayant reçu ainsi des doses importantes au niveau des seins [20]. En Suède, une cohorte de 35000 personnes ayant subi une scintigraphie thyroïdienne à l'iode 131 a été étudiée $[18,19]$.

\section{I.3. Expositions professionnelles}

Plusieurs groupes de travailleurs ont été exposés à des doses importantes sur des périodes prolongées alors que les dangers de l'exposition aux rayonnements ionisants restaient méconnus [36] : peintres de cadrans luminescents, anciens radiologues et techniciens de radiologie [29], mineurs travaillant dans un environnement à forte teneur en radon.

D'autres enquêtes ont été entreprises ces dernières années parmi des travailleurs de l'industrie nucléaire $[2,16,33,40]$.

\subsection{Expositions chroniques de populations}

Certaines populations exposées de façon chronique à de faibles doses de radiations ont également été étudiées: personnes exposées à des retombées radioactives d'essais nucléaires [27], personnes vivant dans des habitations à forte teneur en radon ou dans des régions où l'irradiation naturelle est notablement élevée $[10,14,35]$.

\section{MOYENS D'ÉVALUATION DU RISQUE RADIOCANCÉRIGĖNE}

\section{II.1. Relation dose-effet}

L'étude de la relation entre la dose absorbée et la réponse observée (en l'occurence le taux de cancers) chez divers individus exposés donne une première idée du risque. Mais des difficultés existent pour l'évaluation du risque de cancer aux faibles doses et faibles débits de dose. En effet, dans ce cas, le risque lié aux irradiations devient tellement minime, comparativement au risque naturel de cancer, qu'il devient impossible à individualiser, même dans de très grands groupes de populations $[32,34]$. 
On va donc extrapoler aux faibles doses et faibles débits de dose, les risques connus pour les fortes doses, en utilisant différents modèles mathématiques pour la relation entre la dose et l'effet observé.

Le modèle le premier propósé et très largement utilisé a été celui d'une relation linéaire sans seuil [11]. II était considéré comme une estimation prudente, ayant peu de chance de sous-estimer les risques aux faibles doses et offrait une grande simplicité. Les recommandations de la Commission internationale de protection radiologique (CIPR), à la base de l'actuelle réglementation en matière de radioprotection dans le monde entier, se sont basées sur ce modèle [9]. Ultérieurement, d'autres modèles, en particulier quadratique ou linéaire-quadratique, ont été utilisés, considérant une efficacité moindre des faibles doses comparativement aux fortes doses [12].

Enfin, la courbe dose-effet peut prendre un aspect insolite aux très fortes doses : l'incidence de la plupart des cancers en expérimentation animale et de certains cancers chez l'homme ne croît pas indéfiniment avec la dose. A partir d'une certaine dose, on observe un plateau ou même une incurvation vers le bas, que l'on explique par des phénomènes de mort cellulaire. Certains modèles mathématiques de courbe dose-réponse en tiennent compte.

\section{II.2. Facteurs de risque}

On exprime traditionnellement le risque associé à l'exposition de deux manières:

(1) Le risque absolu est indépendant de l'incidence naturelle du risque et ne tient compte que des cas excédentaires. Le coefficient de risque absolu correspond à la différence entre le nombre de cas observés et le nombre de cas attendus, par référence à une population comparable mais non irradiée. En général, on s'intéresse à l'excès de décès par cancers que l'on rapporte à l'unité d'exposition (le gray) et à l'unité de temps (l'année - a) dans la population étudiée (rapportée à un nombre défini de personnes - $P$ - par exemple 1000). Ainsi, le risque absolu est caractérisé par le nombre de décès excédentaires pour $10^{3}$ PaGy.

(2) Le risque relatif, au contraire, est directement lié à l'incidence naturelle du risque, en l'occurence du risque de mortalité par cancer. Le coefficient de risque relatif correspond à un facteur multiplicatif du risque spontané. On l'obtient en divisant le nombre de cas observés dans la population exposée par le nombre de cas prévus dans une population contrôle.

Quel que soit le modèle utilisé, la mesure du risque est basée sur des données observées (nombre de décès par cancer). Le coefficient de risque peut être modulé en fonction de diverses variables, telles le sexe, l'âge à l'exposition et le temps écoulé depuis l'exposition. 


\section{II.3. Modèles de projection de risque}

En raison de la très longue période de latence des cancers radioinduits, tous les cancers en excès n'ont pas encore été observés dans les populations irradiées étudiées. Or, il est important, du point de vue de la santé publique et de la réglementation, de connaître le plus précisément possible l'impact de l'exposition aux rayonnements sur une population. Comme nous ne disposons de données que sur des fractions de la vie des individus exposés, il est nécessaire de projeter les risques sur leur vie entière. Ces projections de risque dépendent fortement des risques réels déjà observés, mais aussi du modèle de projection utilisé.

De même que les coefficients de risque peuvent être exprimés de deux façons différentes, on utilise deux modèles pour les projections de risque au-delà de la période d'observation:

(1) Le modèle additif ou modèle de risque absolu si l'on considère que le risque excédentaire reste constant et indépendant de l'incidence spontanée du cancer considéré;

(2) Le modèle multiplicatif ou modèle de risque relatif lorsque le risque reste une fraction constante du risque naturel de cancer. risque :

Différentes données sont indispensables au calcul des projections de

1. Période de latence et durée de plateau : les leucémies ont une phase de latence minimale de 2 à 5 ans, les tumeurs solides de 10 ans; pour les leucémies, le risque excédentaire diminue au bout de 10 ans, mais persiste à un faible niveau jusqu'à 40 ans après l'exposition; pour les autres cancers, la période de plateau paraît indéterminée; dans une seule étude, celle des spondylarthrites, le risque paraît diminuer 25 ans après l'exposition;

2. Taux de mortalité générale et de mortalité pour différents types de cancers dans la population de référence non exposée;

3. Composition par âge et par sexe de la population exposée, en tenant compte de l'âge au moment de l'exposition et du temps écoulé depuis;

4. Forme retenue pour la courbe dose-effet ;

5. Coefficients de risque de mortalité par cancer calculés selon le modèle de risque absolu ou de risque relatif;

6. Prise en compte de certains facteurs environnementaux, par exemple le tabac pour le cancer du poumon.

Ces modèles de projection de risque permettent de calculer des indices de détriment sanitaire sur la vie entière, à savoir:

- le nombre de décès excédentaires,

- ou le nombre d'années de vie perdues exprimé pour 1000 personnes exposées à une irradiation d'1 Gy. 


\section{II.4. Les incertitudes}

L'évaluation des risques cancérigènes des rayonnements, comme nous l'avons vu, ne repose pas uniquement sur l'observation, en raison de données insuffisantes pour les faibles doses et de périodes de suivi trop courtes dans la plupart des grandes enquêtes. II s'agit donc d'estimations, variables selon le modèle mathématique choisi pour l'extrapolation du risque.

Indépendamment de ces incertitudes tenant au manque d'informations directes, certaines données de base, comme la dosimétrie, restent parfois imprécises. Enfin, se pose le problème de la transposition du risque estimé à d'autres populations, le plus souvent exposées à une irradiation fort différente de celle pour laquelle le risque a été calculé [1].

\section{II.4.1. Incertitudes liées aux modèles mathématiques}

En l'état actuel de nos connaissances, il reste impossible d'opter pour un modèle préférentiellement à un autre, aussi bien pour la forme de la relation dose-effet que pour les projections de risque. Une meilleure approche biologique des mécanismes de la radiocancérogenèse paraît indispensable pour appréhender au mieux ces modèles.

- La relation dose-effet est probablement différente selon le tissu considéré. Ainsi, dans l'étude LSS des survivants d'Hiroshima et Nagasaki couvrant les années 1950 à 1985 [37], SHIMIZU et al. ont étudié (avec la dosimétrie DS 86) la forme de la courbe dose-réponse qui s'adaptait le mieux aux données, pour la leucémie, pour tous les cancers sauf leucémie et pour quatre autres sites spécifiques de cancer (colon-estomac-poumon et sein chez la femme). Dans tous les cas, sauf le cancer du colon, le modèle linéaire simple s'adapte aussi bien que le modèle linéaire quadratique, et le modèle quadratique est moins bon. Pour le cancer du colon, modèles quadratique et linéaire quadratique sont équivalents et supérieurs au modèle linéaire simple. Enfin, pour la leucémie, l'ajustement s'améliore lorsque l'on fait intervenir une composante de mort cellulaire aux fortes doses et le meilleur modèle devient alors le modèle linéaire quadratique; de la même façon, si l'on ne tient compte que des leucémies qui avaient reçu une dose de moins de $4 \mathrm{~Gy}$, le modèle linéaire quadratique est meilleur que le modèle linéaire simple. STRAUME et MOORE, se basant également sur les données récentes d'Hiroshima et Nagasaki, ont recherché la forme de la courbe dose-réponse qui s'ajustait le mieux [41]; la forme de la courbe semble principalement dépendre de l'âge à l'exposition. Le modèle quadratique ou linéaire quadratique est meilleur pour les personnes exposées à un âge plus jeune et le modèle linéaire pour celles exposées à un âge plus avancé.

- Les deux modèles de projection de risque proposés ne s'adaptent pas non plus parfaitement aux données [7]. Ainsi, MUIRHEAD et DARBY ont essayé de déterminer lequel des modèles de risque relatif ou de risque absolu s'ajustait le mieux aux données de mortalité par cancer (sauf leucémie) chez les survivants d'Hiroshima [30, 31]. Ils ont proposé un 
modèle statistique général pour la projection du risque et ont vérifié l'ajustement de ce modèle aux données disponibles, les modèles additif et multiplicatif en constituant des cas spéciaux. Lorsque le risque est exprimé en intégrant diverses covariables, on se rapproche plus de l'un ou de l'autre modèle, selon le nombre et la nature de ces covariables. Ainsi, lorsque l'on prend en considération l'âge à l'exposition et le sexe, le modèle de risque relatif est meilleur; au contraire, le modèle de risque absolu donne un meilleur ajustement si l'on intègre l'âge à l'exposition et le temps écoulé depuis l'exposition. Les deux modèles sont équivalents quand les trois variables (sexe-âge à l'exposition et temps écoulé depuis l'exposition) sont associées. Par contre, en l'absence de covariable auxiliaire, aucun des deux modèles particuliers de risque relatif ou absolu ne s'ajuste bien aux données. LITTLE et CHARLES ont utilisé un modèle de risque intermédiaire entre les modèles de risque absolu et de risque relatif [26].

\section{I1.4.2. Incertitudes sur les données de bases}

Ces incertitudes concernent la dosimétrie et les données de mortalité par cancer de la population de référence.

- La dosimétrie : la plupart des organismes internationaux ont basé leurs estimations de risque sur l'expérience des survivants des explosions atomiques japonaises et par suite sur les doses absorbées calculèes pour chacun des survivants pris en compte dans l'étude LSS. Or la dosimétrie TD 65 , sur laquelle se fondaient tous les calculs de risque jusqu'à ces dernières années et qui a servi de base à la réglementation en cours, vient d'être révisée et remplacée par la dosimétrie DS 86 . Les principales différences pour la nouvelle dosimétrie sont les suivantes [38] :

- exposition aux neutrons inférieure aux estimations préalables (de $90 \%$ à Hiroshima et d'environ $30 \%$ à Nagasaki) ;

- diminution du facteur de transmission des rayons gamma à travers les murs en bois des maisons japonaises d'environ $50 \%$; ceci conduit à une diminution de la dose en "kerma protégé" ou kerma à l'intérieur des habitations par rapport à l'ancienne dosimétrie;

- augmentation du facteur de transmission des rayons gamma aux différents organes, qui contrebalance la diminution de la transmission des rayonnements dans les habitations. En terme de dose absorbée aux organes, les facteurs de risque sont très comparables dans les deux dosimétries (tableau I).

Même avec cette nouvelle dosimétrie, des inconnues persistent: pour $18 \%$ environ de la cohorte LSS prise en compte dans la dosimétrie TD 65, les doses n'ont pu être jusqu'à présent récapitulées. D'autre part, la bombe qui a explosé à Hiroshima n'a jamais été expérimentée depuis et certains paramètres en sont imparfaitement connus. Enfin, en raison de l'irradiation due aux neutrons, il devient très difficile de déterminer leur efficacité biologique relative (EBR) par rapport aux rayonnements gamma. 
TABLEAU ।

Comparaison entre les dosimétries DS86 et T65DR concernant la mortalité par cancer basée sur la dose absorbée aux organes

\begin{tabular}{|c|c|c|c|c|c|}
\hline $\begin{array}{c}\text { Localisation } \\
\text { du } \\
\text { cancer }\end{array}$ & $\begin{array}{c}\text { Système } \\
\text { dosimétrique }\end{array}$ & $\begin{array}{l}\text { Excès de } \\
\text { risque relatif } \\
/ 1 \text { Gy }\end{array}$ & $\begin{array}{l}\text { DS86/ } \\
\text { T65DR }\end{array}$ & $\begin{array}{l}\text { Excès de } \\
\text { décès } \\
/ 10^{4} \mathrm{PaGy}\end{array}$ & $\begin{array}{l}\text { DS86/ } \\
\text { T65DR }\end{array}$ \\
\hline Leucémie & $\begin{array}{l}\text { DS86 } \\
\text { T65DR }\end{array}$ & $\begin{array}{l}5,21 \\
5,76\end{array}$ & 0,90 & $\begin{array}{l}2,94 \\
3,11\end{array}$ & 0,95 \\
\hline $\begin{array}{l}\text { Tous cancers } \\
\text { sauf } \\
\text { leucémie }\end{array}$ & $\begin{array}{l}\text { DS86 } \\
\text { T65DR }\end{array}$ & $\begin{array}{l}0,41 \\
0,58\end{array}$ & 0,71 & $\begin{array}{l}10,13 \\
13,97\end{array}$ & 0,73 \\
\hline Oesophage & $\begin{array}{l}\text { DS86 } \\
\text { T65DR }\end{array}$ & $\begin{array}{l}0,58 \\
0,67\end{array}$ & 0,87 & $\begin{array}{l}0,45 \\
0,49\end{array}$ & 0,92 \\
\hline Estomac & $\begin{array}{l}\text { DS866 } \\
\text { T65DR }\end{array}$ & $\begin{array}{l}0,27 \\
0,39\end{array}$ & 0,69 & $\begin{array}{l}2,42 \\
3,34\end{array}$ & 0,72 \\
\hline Colon & $\begin{array}{l}\text { DS86 } \\
\text { T65DR }\end{array}$ & $\begin{array}{l}0,85 \\
1,04\end{array}$ & 0,82 & $\begin{array}{l}0,81 \\
0,98\end{array}$ & 0,83 \\
\hline Poumon & $\begin{array}{l}\text { DS86 } \\
\text { T65DR }\end{array}$ & $\begin{array}{l}0,63 \\
0,72\end{array}$ & 0,88 & $\begin{array}{l}1,68 \\
1,89\end{array}$ & 0,89 \\
\hline Sein (femme) & $\begin{array}{l}\text { DS86 } \\
\text { T65DR }\end{array}$ & $\begin{array}{l}1,19 \\
0,91\end{array}$ & 1,31 & $\begin{array}{l}1,20 \\
0,90\end{array}$ & 1,33 \\
\hline Ovaire & $\begin{array}{l}\text { DS86 } \\
\text { T65DR }\end{array}$ & $\begin{array}{l}1,33 \\
1,21\end{array}$ & 1,10 & $\begin{array}{l}0,71 \\
0,64\end{array}$ & 1,11 \\
\hline Vessie & $\begin{array}{l}\text { DS86 } \\
\text { T65DR }\end{array}$ & $\begin{array}{l}1,27 \\
1,59\end{array}$ & 0,80 & $\begin{array}{l}0,66 \\
0,81\end{array}$ & 0,81 \\
\hline $\begin{array}{l}\text { Myélome } \\
\text { multiple }\end{array}$ & $\begin{array}{l}\text { DS86 } \\
\text { T65DR }\end{array}$ & $\begin{array}{l}2,39 \\
2,39\end{array}$ & 0,96 & $\begin{array}{l}0,26 \\
0,29\end{array}$ & 0,90 \\
\hline
\end{tabular}

Or, plus l'EBR des neutrons est élevée, plus la disparité entre les deux dosimétries augmente (tableau II). Dans les deux cas, cependant, le risque est majoré pour une plus petite valeur de l'EBR des neutrons.

Bien, que les différences soient très réduites entre les deux villes et ne soient plus statistiquement significatives avec le nouveau système DS 86 , la mortalité semble plus élevée à Hiroshima qu'à Nagasaki pour quelques sites de cancers dont la leucémie [38]. On a également enregistré une plus grande fréquence d'anomalies chromosomiques et d'effets précoces (comme l'alopécie) à Hiroshima. Ces diverses constatations doivent faire rechercher une explication à ces différençes entre les deux villes, jusqu'à présent attribuées aux différences de la dose neutrons. 
TABLEAU ॥

Comparaison des décès excédentaires $/ 10^{4} \mathrm{PaSv}$ pour différentes valeurs de I'EBR des neutrons en se référant aux doses absorbées aux organes dans les dosimétries DS86 et T65DR

\begin{tabular}{|c|c|c|c|c|}
\hline $\begin{array}{l}\text { Localisation } \\
\text { du cancer }\end{array}$ & EBR & DS86 & T65DR & DS86/T65DR \\
\hline Leucémie & $\begin{array}{r}1 \\
10 \\
20 \\
\end{array}$ & $\begin{array}{l}2,95 \\
2,67 \\
2,40 \\
\end{array}$ & $\begin{array}{l}3,08 \\
1,81 \\
1,23 \\
\end{array}$ & $\begin{array}{l}0,96 \\
1,48 \\
1,95 \\
\end{array}$ \\
\hline $\begin{array}{l}\text { Tous cancers } \\
\text { sauf leucémie }\end{array}$ & $\begin{array}{r}1 \\
10 \\
20\end{array}$ & $\begin{array}{r}10,10 \\
9,41 \\
8,76\end{array}$ & $\begin{array}{r}13,72 \\
8,99 \\
6,34\end{array}$ & $\begin{array}{l}0,73 \\
1,05 \\
1,38\end{array}$ \\
\hline Estomac & $\begin{array}{r}1 \\
10 \\
20 \\
\end{array}$ & $\begin{array}{l}2,63 \\
2,36 \\
2,10 \\
\end{array}$ & $\begin{array}{l}3,38 \\
2,02 \\
1,34 \\
\end{array}$ & $\begin{array}{l}0,78 \\
1,17 \\
1,57 \\
\end{array}$ \\
\hline Colon & $\begin{array}{r}1 \\
10 \\
20\end{array}$ & $\begin{array}{l}0,76 \\
0,73 \\
0,69\end{array}$ & $\begin{array}{l}0,93 \\
0,65 \\
0,49\end{array}$ & $\begin{array}{l}0,82 \\
1,12 \\
1,41\end{array}$ \\
\hline Poumon & $\begin{array}{r}1 \\
10 \\
20\end{array}$ & $\begin{array}{l}1,80 \\
1,59 \\
1,42\end{array}$ & $\begin{array}{l}1,90 \\
1,18 \\
0,80\end{array}$ & $\begin{array}{l}0,95 \\
1,35 \\
1,78\end{array}$ \\
\hline Sein (femme) & $\begin{array}{r}1 \\
10 \\
20\end{array}$ & $\begin{array}{l}1,22 \\
1,00 \\
0,82\end{array}$ & $\begin{array}{l}0,90 \\
0,43 \\
0,26\end{array}$ & $\begin{array}{l}1,36 \\
2,33 \\
3,15\end{array}$ \\
\hline
\end{tabular}

- Les taux de base des cancers: ils se modifient au cours du temps avec le changement rapide des niveaux d'exposition à divers agents cancérigènes. Or, toute population exposée à des rayonnements ionisants est également exposée à d'autres facteurs de risque (en particulier environnementaux tels le tabac, les facteurs alimentaires, les cancérigènes chimiques), lesquels constituent un risque de cancer beaucoup plus important que de faibles doses de radiations.

\section{II.4.3. Incertitudes pour les transpositions de risque à d'autres populations}

L'estimation des coefficients de risque et la projection des risques de la vie durant s'appliquent toujours à une population particulière. Or, des modifications des taux de mortalité de base survenant parallèlement à l'évolution des facteurs cancérigènes sont susceptibles de modifier les projections de risque de cancer la vie durant. En utilisant les coefficients de risque estimés d'après les données des survivants d'Hiroshima et Nagasaki, des projections de risque ont été faites pour trois populations différentes: japonaise, anglaise et portoricaine et les résultats obtenus sont très voisins [44]. Cependant, ces conclusions ne s'appliquent qu'à l'extrapolation concernant les projections de risque et l'on ne sait pas de quelle manière les coefficients de risque eux-mêmes pourraient varier entre différentes populations.

D'autre part, les facteurs de risque de cancer sont calculés pour une population donnée soumise à une irradiation définie, souvent irradiation à 
forte dose et à fort débit de dose. II est peu probable que ces facteurs de risque soient parfaitement transposables à une population exposée à des conditions d'irradiation très différentes, en règle générale faible dose à faible débit de dose.

\section{EVALUATION DU RISQUE CANCÉRIGĖNe PAR L'UNSCEAR 1988 [44]}

\section{III.1. Eléments servant à l'évaluation du risque}

III.1.1. Trois populations irradiées regroupant un nombre très important de personnes suivies pendant de nombreuses années ont été choisies pour évaluer le risque cancérigène des rayonnements. Outre les survivants japonais d'Hiroshima et Nagasaki [37], deux groupes de patients sont considérés: britanniques irradiés pour spondylarthrite ankylosante [13] et plus de $\mathbf{8 0} 000$ femmes dans une étude internationale sur le cancer du col de l'utérus [5]. Le tableau III récapitule les caractéristiques de ces trois études.

\section{TABLEAU III}

Comparaison des principales caractéristiques des études japonaises, de spondylarthrite et de cancer du col de l'utérus

\begin{tabular}{|c|c|c|c|}
\hline & Survivants japonais & Spondylarthrite & Cancer du col \\
\hline Nature de l'étude & prospective & $\begin{array}{l}\text { rétrospective- } \\
\text { prospective }\end{array}$ & $\begin{array}{l}\text { rétrospective- } \\
\text { prospective }\end{array}$ \\
\hline $\begin{array}{l}\text { Taille de } \\
\text { de l'échantillon }\end{array}$ & 76000 & 14000 & 83000 \\
\hline $\begin{array}{l}\text { Répartition } \\
\text { par sexe }\end{array}$ & $F=59 \%$ & $\mathrm{~F}=17 \%$ & $F=100 \%$ \\
\hline $\begin{array}{l}\text { Age à l'irradiation } \\
\text { (années) }\end{array}$ & $0-90$ & $>15$ & $30-70$ \\
\hline $\begin{array}{l}\text { Temps moyen de } \\
\text { suivi (années) }\end{array}$ & 28,8 & 13 & 7,6 \\
\hline Type de contrôle & interne & $\begin{array}{c}\text { population adulte } \\
\text { masculine } \\
\text { UK }\end{array}$ & $\begin{array}{l}\text {. population adulte } \\
\text { féminine UK } \\
\text { et contrôle interne }\end{array}$ \\
\hline $\begin{array}{l}\text { Nature de } \\
\text { la dosimétrie }\end{array}$ & $\begin{array}{l}\text { individuelle } \\
\text { (DS 86) }\end{array}$ & $\begin{array}{l}\text {. individuelle pour } \\
\text { leucémie } \\
\text { dose moyenne sur } \\
\text { échantillon pour } \\
\text { les autres cancers }\end{array}$ & $\begin{array}{l}\text { dose moyenne } \\
\text { sur échantillon } \\
\text { (étude de } \\
\text { cohorte) } \\
\text {. individuelle } \\
\text { (étude cas- } \\
\text { témoin) }\end{array}$ \\
\hline $\begin{array}{l}\text { Nature de } \\
\text { l'irradiation }\end{array}$ & instantanée corps entier & fractionnée/localisée & chronique/localisée \\
\hline $\begin{array}{l}\text { Dose moyenne } \\
\text { (Gy) } \\
\text { Limites de doses } \\
\text { (Gy) }\end{array}$ & $\begin{array}{c}0,24 \\
(0,01-6)\end{array}$ & $\begin{array}{c}1,9 \\
(0-8,06) \\
\end{array}$ & très variable \\
\hline $\begin{array}{l}\text { Personnes- } \\
\text { années à risque }\end{array}$ & 2185000 & 184000 & 623800 \\
\hline
\end{tabular}


III.1.2. La détermination du risque repose sur la mortalité liée aux cancers et non sur l'incidence des cancers. Les leucémies d'une part, tous les autres cancers d'autre part, ont été pris en compte. Enfin, le risque a également été examiné pour huit cancers particuliers reconnus comme pouvant être radioinduits : vessie, sein chez la femme, colon, poumon, ovaire, œsophage, estomac et myélome multiple.

III.1.3. L'UNSCEAR a utilisé des coefficients de risque de décès par cancer, applicables pour les fortes doses et forts débits de dose.

- Dans le cas des spondylarthrites et des cancers du col, ces coefficients sont ceux communiqués par les auteurs.

- Pour la population d'Hiroshima et Nagasaki, ils sont basés sur la nouvelle dosimétrie DS 86 et reposent sur un modèle de risque relatif linéaire utilisant la dose absorbée aux organes. Pour l'estimation, l'EBR des neutrons a été considérée comme égale à 1 . Les coefficients représentent des valeurs moyennes pour les deux villes, les deux sexes et tous les âges au moment de l'irradiation.

III.1.4. Des projections de risque sur la vie entière ont été réalisées selon les deux types de modèles, additif ou multiplicatif. On a considéré la latence minimale à 2 ans pour les leucémies, 10 ans pour les autres cancers, et le plateau à 40 ans et durant toute la vie, respectivement pour les leucémies et les tumeurs solides.

Le détriment a été exprimé de deux façons:

- soit mortalité excédentaire par cancer sur toute la vie,

- soit perte d'espérance de vie en personnes-années, et ces valeurs ont été rapportées à. 1000 personnes exposées à 1 Gy d'irradiation à fort débit de dose.

Les calculs ont été effectués pour la population dans son ensemble ainsi que la population au travail (entre 25 et 64 ans).

\section{III.2. Coefficients de risque proposés par I'UNSCEAR 1988 (tableau IV)}

Les coefficients de risque moindre observés dans les populations de malades pourraient s'expliquer par la nature différente de l'irradiation, car le débit de dose était très élevé seulement à Hiroshima et Nagasaki.

Ces coefficients de risque sont moins élevés pour la population japonaise lorsque l'on exclut les personnes âgées de moins de 25 ans au moment de l'irradiation. 
D. HUBERT

TABLEAU IV

Etude comparative de l'excédent de risque relatif pour une dose absorbée d'1Gy par organe (pour les rayonnements à faible TLE et à fort débit de dose)

\begin{tabular}{|c|c|c|c|c|}
\hline & $\begin{array}{c}\text { Surviv } \\
>25 \text { ans }\end{array}$ & $\begin{array}{l}\text { ants japonais } \\
\text { tous âges ATB* }\end{array}$ & Spondylarthrite & Cancer du col \\
\hline Leucémie & 3,8 & 5,21 & 3,50 & 088 \\
\hline $\begin{array}{l}\text { Tumeurs } \\
\text { solides }\end{array}$ & 0,35 & 0,41 & 0,14 & - \\
\hline Vessie & & 1,27 & 0,19 & 0,07 \\
\hline Sein & & 1,19 & - & 0,03 \\
\hline Rein & & 0,58 & 0,12 & 0,71 \\
\hline Colon & & 0,85 & - & 0 \\
\hline Larynx & & 0,51 & 0,15 & - \\
\hline Poumon & & 0,63 & 0,13 & - \\
\hline $\begin{array}{l}\text { Myélome } \\
\text { multiple }\end{array}$ & & 2,29 & - & - \\
\hline Oesophage & & 0,58 & 0,29 & - \\
\hline Ovaire & & 1,33 & 0 & 0,01 \\
\hline Rectum & & 0 & 0,03 & 0,02 \\
\hline Estomac & & 0,27 & 0,004 & 0,69 \\
\hline
\end{tabular}

Risque absolu (décès excédentaires $/ 10^{4} \mathrm{PaGy}$ )

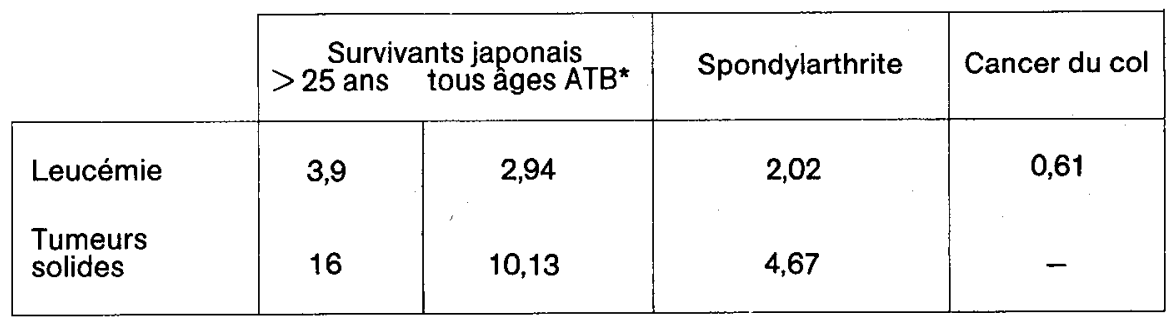

* ATB = à l'époque de l'explosion atomique. 


\section{III.3. Projections de risque proposées par I'UNSCEAR 1988}

Le tableau $V$ récapitule pour la population adulte les projections de risque effectuées dans les trois études mentionnées. Là encore, les évaluations effectuées pour la spondylarthrite sont de 4 à 1,5 fois moindres que celles s'appliquant aux irradiés des bombardements atomiques.

\section{TABLEAU $V$}

Etude comparative d'excédent de mortalité et de réduction d'espérance de vie, la vie durant (pour 1000 adultes exposés à 1 Gy par organe pour un rayonnement à faible TEL et à fort débit de dose)

\begin{tabular}{|c|c|c|c|c|c|c|}
\hline & \multicolumn{2}{|c|}{$\begin{array}{l}\text { Etude } \\
\text { japonaise }\end{array}$} & \multicolumn{2}{|c|}{ Spondylarthrite } & \multicolumn{2}{|c|}{ Cancer du col } \\
\hline & $\begin{array}{l}\text { Multi- } \\
\text { plicatif }\end{array}$ & Additif & $\begin{array}{l}\text { Multi- } \\
\text { plicatif }\end{array}$ & Additif & $\begin{array}{l}\text { Multi- } \\
\text { plicatif }\end{array}$ & Additif \\
\hline \multicolumn{7}{|c|}{ EXCÉDENT EN DÉCĖS } \\
\hline \multirow{3}{*}{ Leucémie $^{M}{ }_{\text {moyenne }}{ }^{a}$} & 9 & 13 & 14 & 4,4 & - & - \\
\hline & 8,1 & 7 & - & - & 2,8 & 1,4 \\
\hline & 8,6 & 10 & & & & \\
\hline \multirow{3}{*}{$\begin{array}{l}\text { Tumeurs }_{\text {solides }} \mathrm{M} \\
\text { moyenne } \\
\mathrm{b}\end{array}$} & 41 & 30 & 23 & 7,8 & - & - \\
\hline & 52 & 42 & - & - & - & - \\
\hline & 47 & 36 & & & & \\
\hline TOTAL & 56 & 466 & 37 & 12 & & \\
\hline \multicolumn{7}{|c|}{$\begin{array}{l}\text { RÉDUCTION D'ESPÉRANCE DE VIE } \\
\text { (en personnes-années) }\end{array}$} \\
\hline \multirow{3}{*}{${ }_{\text {Leucémie }}^{M}$} & 140 & 290 & 140 & 79 & - & - \\
\hline & 120 & 170 & - & - & 31 & 28 \\
\hline & 130 & 230 & & & & \\
\hline \multirow{3}{*}{$\begin{array}{l}\text { Tumeurs } \\
\text { solides } F \\
\text { moyenne }\end{array}$} & 420 & 510 & 210 & 120 & - & - \\
\hline & 570 & 710 & - & - & - & - \\
\hline & 490 & 610 & & & & \\
\hline TOTAL & 620 & 840 & 350 & 200 & & \\
\hline
\end{tabular}

b) plateau $=$ toute la vie, latence $=10$ ans

Seule, la population japonaise a permis des projections de risque en considérant la population dans son ensemble et pas seulement la population adulte lors de l'irradiation. Les résultats varient notablement, non seulement selon le modèle de projection utilisé, mais aussi selon que l'on utilise des coefficients de risque constants ou ajustés à l'âge (tableau Vl). 


\section{HUBERT}

TABLEAU VI

Excédent de mortalité et réduction d'espérance de vie (pour une population de 1000 personnes de tous âges exposées à $1 \mathrm{~Gy}$ par organe pour un rayonnement à faible TEL et à fort débit de dose)

\begin{tabular}{|c|c|c|c|c|}
\hline & \multicolumn{2}{|c|}{$\begin{array}{l}\text { Coefficient de risque } \\
\text { ajusté à l'âge }\end{array}$} & \multicolumn{2}{|c|}{$\begin{array}{l}\text { Coefficient de risque } \\
\text { constant }\end{array}$} \\
\hline . & Multiplicatif & Additif & Multiplicatif & Additif \\
\hline \multicolumn{5}{|c|}{ EXCÉDENT EN DÉCÉS } \\
\hline $\begin{array}{l}\text { Leucémies } \\
\text { Tumeurs solides }\end{array}$ & $\begin{array}{l}10 \\
97\end{array}$ & $\begin{array}{l}10 \\
32\end{array}$ & $\begin{array}{c}9,7 \\
61\end{array}$ & $\begin{array}{l}9,3 \\
36\end{array}$ \\
\hline TOTAL & 107 & 42 & 71 & 45 \\
\hline \multicolumn{5}{|c|}{$\begin{array}{l}\text { RÉDUCTION D'ESPÉRANCE DE VIE } \\
\text { (en personnes-années) }\end{array}$} \\
\hline $\begin{array}{l}\text { Leucémies } \\
\text { Tumeurs solides }\end{array}$ & $\begin{array}{r}260 \\
1110\end{array}$ & $\begin{array}{l}300 \\
650\end{array}$ & $\begin{array}{l}220 \\
730\end{array}$ & $\begin{array}{l}300 \\
910\end{array}$ \\
\hline TOTAL & 1370 & 950 & 950 & 1210 \\
\hline
\end{tabular}

Enfin, dans le cas de la population japonaise, des projections de risque ont été établies pour différents types de cancers (tableau VII).

TABLEAU VII

Nombre de décès supplémentaires et perte d'espérance de vie par cancer pour 1000 personnes des deux sexes, tous âges confondus, après une exposition d'1Gy (à débit de dose élevé pour des rayonnements de faible TEL et à fort débit de dose). Les calculs sont effectués avec des coefficients de risque moyen quel que soit l'âge et pour la population japonaise.

\begin{tabular}{|l|c|c|c|c|}
\cline { 2 - 5 } \multicolumn{1}{c|}{} & \multicolumn{2}{c|}{ Décès supplémentaires } & \multicolumn{2}{c|}{ Perte d'espérance de vie (années) } \\
\cline { 2 - 5 } & Multiplicatif & Additif & Multiplicatif & Additif \\
\hline Moelle osseuse & 9,7 & 9,3 & 220 & 300 \\
\hline $\begin{array}{l}\text { Tous cancers } \\
\text { (sauf leucémies) }\end{array}$ & 61 & 36 & 730 & 910 \\
\hline Vessie & 3,9 & 2,3 & 30 & 40 \\
\hline Sein & 6 & 4,3 & 110 & 110 \\
\hline Colon & 7,9 & 2,9 & 90 & 70 \\
\hline Poumon & 15,1 & 5,9 & 170 & 150 \\
\hline Myélome multiple & 2,2 & 0,9 & 30 & 20 \\
\hline Ovaire & 3,1 & 2,6 & 60 & 70 \\
\hline Oesophage & 3,4 & 1,6 & 40 & 40 \\
\hline Estomac & 12,6 & 8,6 & 150 & 220 \\
\hline Autres & 11,4 & 6,9 & 140 & 280 \\
\hline TOTAL & 70,7 & 45,3 & 950 & 1200 \\
\hline
\end{tabular}




\section{III.4. Conclusions de l'UNSCEAR 1988 pour les fortes doses et forts débits de dose}

L'UNSCEAR 1988 dans sa conclusion retient un risque de mortalité par radio-cancer sur la vie entière compris entre 4 et $11.10^{-2} \mathrm{~Gy}^{-1}$. Cette estimation provient exclusivement des résultats des survivants d'Hiroshima et Nagasaki irradiés à très fort débit de dose. Bien que l'UNSCEAR ait pris en compte, dans son analyse, d'autres groupes irradiés, leurs valeurs de risques ne sont pas considérées dans les conclusions du rapport. Or, si l’on inclut le groupe de spondylarthrites pour lequel les calculs sont disponibles (risque compris entre 1,2 et $3,7.10^{-2} \mathrm{~Gy}^{-1}$ ), la fourchette de risque de mortalité s'élargit vers le bas et devient comprise entre 1,2 et $11.10^{-2} \mathrm{~Gy}^{-1}$.

Les variations du nombre estimé de décès supplémentaires pour un même groupe sont liées aux différents modèles utilisés, le modèle multiplicatif accroissant le risque. Par contre, lorsque l'on considère les pertes d'espérance de vie, les estimations deviennent comparables pour les deux modèles additif et multiplicatif (tableau VI). Ceci est lié au fait qu'une grande partie des décès projetés surviennent chez des gens très âgés et n'entraînent, par conséquent, qu'une faible diminution de l'espérance de vie.

Enfin, dans le cas de la population japonaise, les résultats sont très influencés par le fait que les enfants sont particulièrement radiosensibles. Si l'on ne considère que le groupe d'adultes, les limites de risque se réduisent entre 4,6 et $5,6 \cdot 10^{-2} \mathrm{~Gy}^{-1}$. En considérant également la population des spondylarthrites, le risque de mortalité par cancer lié aux radiations est alors compris entre 1,2 et $5,6.10^{-2} \mathrm{~Gy}^{-1}$ pour les adultes irradiés à fort débit de dose.

\section{CAS PARTICULIER DES FAIBLES DOSES}

L'UNSCEAR 1988 considère que les évaluations de risque proposées ne s'appliquent que pour des doses comprises entre 0,5 et 6 Gy. Pour des doses plus faibles et de faibles débits de dose, il existe des arguments en faveur d'une non-linéarité de la relation dose-effet. L'UNSCEAR propose, par conséquent, un facteur de réduction permettant d'adapter les facteurs de risque précédemment proposés aux faibles doses et faibles débits de dose. Une grande marge d'incertitude persiste quant à ce facteur de réduction qui demeure compris entre 2 et 10.

Certaines données récentes plaident en faveur d'un effet réduit des faibles doses [24]:

- Le rapport LSS sur la mortalité par cancer chez les survivants japonais de 1950 à 1985 [37] ne signale aucune augmentation des cancers détectable pour des doses inférieures à 0,2 Gy. D'autre part, les données sur la leucémie suggèrent une relation dose-effet curvi-linéaire avec un risque relatif nettement inférieur pour les individus exposés à des doses inférieures à 0,5 Gy. 
- HOLM a étudié 35000 personnes ayant subi une scintigraphie thyroïdienne à l'iode $131[18,19]$. II n'a pas observé d'excès significatif de cancer de la thyroïde. II calcule l'excès de cancers thyroïdiens attendus dans cette population à partir de coefficients de risque de cancer de la thyroïde après irradiation externe par rayons $X$ ou gamma (déterminés en 1985 par le National institute of health), en considérant une dose moyenne à la thyroïde de $0,5 \mathrm{~Gy}$. Comparant le nombre de cancers de la thyroïde attendus à la suite d'une irradiation $X$ ou gamma au nombre de cancers qu'il a réellément observés, il en déduit que le pouvoir cancérogène d'une irradiation interne par les particules bêta de l'iode 131 pourrait être 4 fois moindre que celui d'une irradiation externe par rayons $X$ ou gamma [18]. Une faible augmentation des leucémies a été observée, mais uniquement dans la période de 5 à 9 ans après l'irradiation, et portant aussi bien sur la leucémie lymphoïde chronique, réputée non radioinduite, que sur les autres leucémies [19].

- L'étude de HOWE de cancers du sein secondaires à des radioscopies thoraciques, où l'irradiation avait été extrêmement fractionnée, montre une courbe dose-réponse quadratique ou linéaire quadratique. Le facteur de réduction est supérieur ou égal à 3 [20].

- Aucun taux de cancer excédentaire n'a pu être observé parmi les populations exposées à des taux élevés d'irradiation naturelle, en particulier pour plusieurs études effectuées en Chine.

- Plusieurs enquêtes chez des travailleurs de l'industrie nucléaire n'ont pu déceler d'excès significatif de cancer $[2,16,33,40]$.

- Enfin, en fonction des données disponibles pour l'irradiation chronique par les particules alpha, et tout spécialement le radon, aucun effet n'est décelé dans la gamme des faibles doses. Le problème de l'extrapolation des mineurs vers le public se heurte à des difficultés non résolues [10]. D'ailleurs, DOUSSET souligne que les coefficients de risque pour le cancer du poumon après exposition à de fortes concentrations en radon ne sont pas applicables à la population générale exposée de façon chronique au radon dans les habitations [14].

\section{COMPARAISON AVEC LES ÉTUDES PRÉCÉDENTES}

Plusieurs organismes scientifiques internationaux ont tenté précédemment de quantifier le risque des radiations ionisantes. Ces estimations sont désormais considérées comme inexactes, car fondées sur l'ancienne dosimétrie d'Hiroshima et Nagasaki et sur une période d'observation plus courte des populations irradiées. Néanmoins, elles ont servi à l'établissement des normes de radioprotection en vigueur actuellement et il est intéressant de les comparer aux estimations désormais proposées par I'UNSCEAR 1988.

- En 1977, ce même comité UNSCEAR évaluait le risque de cancer sur toute la vie à $2,5.10^{-2} \mathrm{~Gy}^{-1}$ pour les radiations à fortes doses, en se basant sur un modèle de projection de risque de type additif [43]. Le risque 
de leucémie représentait environ $1 / 5$ du risque total. Enfin, pour les faibles doses, ce risque était réduit à $1.10^{-2} \mathrm{~Gy}^{-1}$.

- Le BEIR III en 1980 a utilisé aussi bien les modèles de projection additif que multiplicatif et a établi des valeurs de risque de faibles doses pour les différents modèles de courbe dose-réponse [12] (tableau VIII).

TABLEAU VIII

Risque de cancers mortels sur la vie après une exposition unique à 0,1 Gy (BEIR III)

\begin{tabular}{|l|c|c|}
\hline \multirow{2}{*}{$\begin{array}{c}\text { Modèle dose/ } \\
\text { réponse }\end{array}$} & \multicolumn{2}{|c|}{ Décès excèdentaires/10 $0^{3}$ personnes/Gy } \\
\cline { 2 - 3 } & $\begin{array}{c}\text { Modèle de projection } \\
\text { multiplicatif }\end{array}$ & $\begin{array}{c}\text { Modèle de projection } \\
\text { additif }\end{array}$ \\
\hline Linéaire & 50,1 & 16,7 \\
Linéaire-quadratique & 22,6 & 7,7 \\
Quadratique & 2,8 & 1 \\
\hline
\end{tabular}

Même en ne tenant pas compte de la possibilité d'une courbe doseréponse quadratique aux faibles doses, les estimations du BEIR III donnent une fourchette de risque comprise entre 0,7 et $5.10^{-2} \mathrm{~Gy}^{-1}$, l'importance des incertitudes restant liée à la méconnaissance des modèles les mieux adaptés.

- En 1985, la Nuclear regulatory commission des Etats-Unis (NUREG) a également fourni des limites supérieure et inférieure de risques d'irradiation à faibles doses et à faibles débits de dose [15]. Elle a pour cela utilisé les deux modèles de projection de risque et des courbes dose-effet linéaires ou linéaires-quadratiques. Le nombre de décès par cancer sur la vie est compris entre 0,3 et $5,7.10^{-2} \mathrm{~Gy}^{-1}$.

- Enfin, le National institute of health (USA) donnait une valeur de $2.10^{-2}$ $\mathrm{Gy}^{-1}$ pour les faibles doses [42].

- Le tableau IX récapitule les estimations de risque de ces différents organismes en les comparant à celles de I'UNSCEAR 1988.

TABLEAU IX

Evaluation comparative de risque de décès par cancer sur la vie pour les faibles doses et faibles débits de dose

\begin{tabular}{|l|c|}
\cline { 2 - 2 } \multicolumn{1}{c|}{} & Décès excédentaires $/ 10^{3}$ personnes/Gy \\
\hline UNSCEAR 1977 & 10 \\
BEIR 1980 & 7 à 50 \\
NUREG 1985 & 3 à 57 \\
NIH 1985 & 20 \\
UNSCEAR 1988* & 4 à 55 \\
\hline
\end{tabular}

* En appliquant le facteur de réduction 2 à 10 au risque des fortes doses estimé de 40 à $110 \cdot 10^{-3}$. $\mathrm{Gy}^{-1}$. 


\section{CONCLUSION}

Le récent rapport de l'UNSCEAR propose une réévaluation d'un facteur 1.6 à 4.4 de ses précédentes estimations de risque pour les fortes doses d'irradiation à débits de dose élevés. Néanmoins, un facteur de réduction de 2 à 10 reste applicable pour les faibles doses et faibles débits de dose. II en résulte donc, pour les conditions d'irradiations qui intéressent la radioprotection des travailleurs aussi bien que du public, des valeurs comparables à celles fournies par d'autres organismes (tableau IX) et compatibles avec la valeur de $1,25.10^{-2}$. Gy ${ }^{-1}$ jusqu'à présent retenue par la CIPR pour élaborer ses recommandations [9].

En raison des nombreuses incertitudes persistantes et principalement liées au type de modèle mathématique utilisé pour la projection de risque sur la vie ou l'extrapolation aux faibles doses et faibles débits de doses, il ne paraît pas actuellement justifié de modifier les normes de radioprotection [6, 24]. Dans les années à venir, la prolongation de la période d'observation des populations à risque et de nouvelles études devraient permettre de réduire la marge d'incertitude et d'évaluer les risques des rayonnements de façon plus précise. II ne faut pas non plus oublier que la majeure partie des doses reçues par la population provient des examens radiologiques médicaux et du radon dans les habitations [8], l'exposition professionnelle intervenant pour moins de $1 \%$ de la dose collective globale. Par conséquent, si l'on admet un risque pour les faibles doses, un effort prioritaire devrait être entrepris concernant la réduction de l'exposition liée au radon dans les habitations et aux radiographies médicales.

\section{BIBLIOGRAPHIE}

[1] ADELSTEIN S.J. - Incertitudes et risques relatifs concernant l'exposition aux radiations. JAMA, 1988, 13, 116-120.

[2] BERAL V., INSKIP H., FRASER P., BOOTH M., COLEMAN D., ROSE G. - Mortality of employees of the United Kingdom Atomic energy authority, 1946-1979. Br. Med. J., $1985,291,440-447$.

[3] BOICE J.D., BLETTNER M., KLEINERMAN R.A., STOVALL M., MOLONEY W.C., ENGHOLM G., AUSTIN D.F., BOSCH A., COOKFAIR D.L., KREMENTZ E.T., LATOURETTE H.B., PETERS L.J., SCHULZ M.D., LUNDELL M., PETTERSSON F., STORM H.H., BELL C.M.J., COLEMAN M.P., FRASER P., PALMER M., PRIOR P., CHOI N.W., HISLOP T.G., KOCH M., ROBB D., ROBSON D., SPENGLER R.F., VON FOURNIER D., FRISCHKORN R., LOCHMULLER H., POMPE-KIRN V., RIMPELA A., KJORSTAD K., PEJOVIC M.H., SIGURDSSON K., PISANI P., KUCERA H., HUTCHISON G.B. - Radiation dose and leukemia risk in patients treated for cancer of the cervix. J. Natl. Cancer Inst., 1987, 79, 1295-1311.

[4] BOICE J.D., DAY N.E., ANDERSEN A., BRINTON L.A., BROWN R., CHOI N.W., CLARKE E.A., COLEMAN M.P., CURTIS R.E., FLANNERY J.T., HAKAMA M., HAKULINEN T., HOWE G.R., JENSEN O.M., KLEINERMAN R.A., MAGNIN D., MAGNUS K., MAKELA K., MALKER B., MILLER A.B., NESON N., PATTERSON C.C., PETTERSSON F., POMPE-KIRN V., PRIMIC-ZAKELJ M., PRIOR P., RAVNIHAR B., SKEET R.G., SKERVEN J.E., SMITH P.G., SOK K., SPENGLER R.F., STORM H.H., STOVALL M., TOMKINS G.W.O., WALL C. - Second cancers following radiation treatment for cervical cancer, an international collaboration among cancer registries. J. Natl. Cancer Inst., 1985, 74, 955-975. 
[5] BOICE J.D., ENGHOLM G., KLEINERMAN R.A., BLETTNER M., STOVALL M., LISCO H., MOLONEY W.C., AUSTIN D.F., BOSCH' A., COOKFAIR D.L., KREMENTZ̈ E.T., LATOURETTE H.B., MERRILL J.A., PETERS L.J., SCHULZ M.D., STORM H.H., BJORKHOLM E., PETTERSSON F., BELL C.M.J., COLEMAN M.P., FRASER P., NEAL F.E., PRIOR P., CHOI N.W., HISLOP T.G., KOCH M., KREIGER N., ROBB D., ROBSON D., THOMSON D.H., LOCHMULLER $H_{\text {., }}$ VON FOURNIER D., FRISCHKORN R., KJORSTAD K.E., RIMPELA A., PEJOVIC M.H., POMPE-KIRN V., STANKUSOVA $H$., BERRINO $F$., SIGURDSSON $K$., HUTCHINSON G.B., MAC MAHON B. - Radiation dose and second cancer risk in patients treated for cancer of the cervix. Radiat. Res., 1988, 116, 3-55.

[6] CHAU N.P. - Radiation carcinogenesis in humans: is it necessary to revise exposure dose limits based on recent estimates of lifetime risks? Health Phys., 1987, $52,753-761$.

[7] CHAU N.P., GUIGUET M. - Radiation carcinogenesis : cumulative empirical distribution functions and risk projection models. Health Phys., 1986, 51, 447-455.

[8] CLARKE R.H., SOUTHWOOD T.R.E. - Risks from ionizing radiation. Nature, 1989, 338, 197-198.

[9] COMMISSION INTERNATIONALE DE PROTECTION RADIOLOGIQUE (CIPR) Recommandations de la Commission internationale de protection radiologique (Publication CIPR 26). Oxford: Pergamon Press, 1977, $63 \mathrm{p}$.

[10] COMMITTEE ON THE BIOLOGICAL EFFECTS OF IONIZING RADIATIONS (BEIR), NATIONAL RESEARCH COUNCIL - Health risks of radon and other internally deposited alpha-emitters: BEIR IV. Washington D.C.: National academy of sciences, 1988, $602 \mathrm{p}$.

[11] COMMITTEE ON THE BIOLOGICAL EFFECTS OF IONIZING RADIATIONS (BEIR), NATIONAL RESEARCH COUNCIL - The effects on populations of exposure to low levels of ionizing radiation : BEIR I, Washington D.C. : National academy of sciences, $1972,217 \mathrm{p}$.

[12] COMMITTEE ON THE BIOLOGICAL EFFECTS OF IONIZING RADIATIONS (BEIR), NATIONAL RESEARCH COUNCIL - The effects on populations of exposure to low levels of ionizing radiation: BEIR III. Washington D.C.: National academy of sciences, 1980, $651 \mathrm{p}$.

[13] DARBY S.C., DOLL R., GILL S.K., SMITH P.G. - Long term mortality after a single treatment course with $\mathrm{X}$-rays in patients treated for ankylosing spondylitis. $\mathrm{Br} . \mathrm{J}$. Cancer, $1987,55,179-190$.

[14] DOUSSET $M$. - Cancer et exposition de l'homme aux rayonnements ionisants d'origine naturelle. In: Cancérogenèse par les faibles doses de radiations ionisantes et normes de sécurité (LATARJET R., Ed.). Paris: Académie des sciences, 1988, 20-23.

[15] GILBERT E. - Late somatic effects. In : Health effects models for nuclear power plant accident consequence analysis' (EVANS J.S. et al, Eds). Rapport NUREG/CR4214, 1985, p. $\|-89$ II-138.

[16] GILBERT E., MARKS. - An analysis of mortality of workers in a nuclear facility. Radiat. Res., 1979, 79, 122-148.

[17] HAMILTON T.E., VAN BELLE G., LOGERFO J.P. - Thyroid neoplasia in Marshall islanders exposed to nuclear fallout. JAMA, 1987, 258, 629-636. 
[18] HOLM L.E., WIKLUND K.E., LUNDELL G.E., BERGMAN N.A., BJENKENGREN G. CEDERQUIST E.S., ERICSSON U.B.C., LARSSON L.G., LIDBËRG M.E., LINDBERG R.S., WICKLUND H.V., BOICE J.D. - Thyroid cancer after diagnostic doses of iodine -131 : a retrospective cohort study. J. Natl. Cancer Inst., 1988, 80, 1132-1138.

[19] HOLM L.E., WIKLUND K.E., LUNDELL G.E., BERGMAN N.A., BJENKENGREN G. ERICSSON U.B.C., CEDERQUUIST E.S., LIDBERG M.E., LINDBERG R.S., WICKLUND $H . V$., BOICE J.D. - Cancer risk in population examined with diagnostic doses of 131 I. J. Natl. Cancer Inst., 1989, 81, 302-306.

[20] HOWE G.R. - Epidemiology of radiogenic breast cancer. In : Radiation carcinogenesis : epidemiology and biological significance (BOICE J.D., FRAUMENI J.F., (Eds.). New York : Raven Press, 1984, 119-129.

[21] ILYN L.A., PAVLOVSKIJ O.A. - Conséquences radiologiques de l'accident de Tchernobyl en Union Soviétique et mesures prises pour en atténuer l'impact. AIEA Bull., 1987, N. 4, 17-24.

[22] JABLON S. - Epidemiologic perspectives in radiation carcinogenesis. In : Radiation carcinogenesis : epidemiology and biological significance (BOICE J.D., FRAUMENI J.F., Eds.). New York : Raven Press, 1984, 1-8.

[23] LAFUMA J. - L'expérimentation animale sur l'effet des iaibles doses. In: Cancérogenèse par les faibles doses de radiations ionisantes et normes de sécurité (LATARJET R., Ed). Paris: Académie des sciences, 1988, 17-19.

[24] LATARJET R., TUBIANA M. - The risks of induced carcinogenesis after irradiation at small doses. The uncertainties which remain after the 1988 UNSCEAR report. Int. J. Radiat. Oncology, 1989, 17, 237-240.

[25] LEWIS C.A., SMITH P.G., STRATTON I.M., DARBY S.C., DOLL R. - Estimated radiation dose to different organs among patients treated for ankylosing spondylitis with a single course of $X$ rays. Brit. J. Radiology, 1988, 61, 212-220.

[26] LITTLE M.P., CHARLES M.W. - Estimation of population cancer risks from the DS86 bomb survivor data. J. Radiol. Prot., 1989, 9, 9-18.

[27] MACHADO S.G., LAND C.E., MCKAY F.W. - Cancer mortality and radioactive fallout in southwestern Utah. Am. J. Epidemiol., 1987, 125, 44-61.

[28] MASSE R. - Irradiation et risque de cancer. Le point de vue du Comité scientifique des Nations Unies; les recherches en cours, Rev. Gén. Nucl., 1989, N. 4. 300-303.

[29] MATANOSKI G.M., STARTWELL P., ELLIOTT E., TONASCIA J., STERNBERG A. Cancer risks in radiologists and radiation workers. In: Radiation carcinogenesis: epidemiology and biological significance (BOICE J.D., FRAUMENI J.F., Eds.). New York : Raven Press, 1984, 83-96.

[30] MUIRHEAD C.R., DARBY S.C. - Distinguishing relative and absolute risk models for radiation-induced cancers. In : Health effects of low dose ionising radiation, London, 11-14 May 1987. Londres : British nuclear energy society, 1988, 45-50.

[31] MUIRHEAD C.R., DARBY S.C. - Modelling the relative and absolute risks of radiation-induced cancers. J.R. Statist. Soc. A., 1987, 150, (part 2) 83-118.

[32] POCHIN E.E. - Difficulties in estimating the effect of low radiation doses in man. J. Radiol. Prot., 1988, 8, 163-171. 
[33] RINSKY R.A., ZUMWALDE R.D., WAXWEILER R.J., MURRAY W.E., BIERBAUM P.J., LANDRIGAN P.J., TERPILAK M., COX C. - Cancer mortality at a naval nuclear shipyard. Lancet, 1981, I, 231-235.

[34] RITENOUR E.R. - Health effects of low level radiation : carcinogenesis, teratogenesis and mutagenesis. Seminars Nuclear Med., 1986, 26, 106-117.

[35] SAMET J.M., NERO A.V. - Indoor radon and lung cancer. N. Engl. J. Med., 1989, $320,591-594$.

[36] SARTWELL P.E., SHORE R.E. - Carcinogenic effects of occupational radiation exposure. In : Radiation carcinogenesis (UPTON A.C., ALBERT R.E. et al, Eds.). New York : Elsevier, 1986, 387-400.

[37] SHIMIZU Y., KATO H., SCHULL W.J. - Life span study report 11, part 2 - Cancer mortality in the years 1950-85 based on the recently revised doses (DS86). Technical report RERF TR5-88, 1988, $102 \mathrm{p}$.

[38] SHIMIZU Y., KATO H., SCHULL W.J., PRESTON D., FUJITA S., PIERCE D. - Life span study report 11, part 1 - Comparison of risk coefficients for site specific cancer mortality based on the DS86 and T65DR shielded kerma and organ doses. Technical report RERF TR12-87, 1987, $61 \mathrm{p}$.

[39] SMITH P.G., DOLL R. - Mortality among patients with ankylosing spondylitis after a single treatment course with X-rays. Brit. Med. J., 1982, 284, 449-460.

[40] SMITH P.G., DOUGLAS A.J. - Mortality of workers at the Sellafield plant of British nuclear fuels. Brit. Med. J., 1986, 293, 845-854.

[41] STRAUME Y., MOORE D. - Shapes of dose-response curves for human cancer: new information from A-bomb data. J. Radiol. Prot., 1988, 8, 157-162.

[42] UNITED STATES NATIONAL INSTITUTES OF HEALTH - Report of the National institutes of health ad hoc working group to develop radioepidemiological tables. Washington D.C.: NIH Publication $\mathrm{n}^{\mathrm{o}} 85-2748,1985,355 \mathrm{p}$.

[43] UNITED NATIONS SCIENTIFIC COMMITTEE ON THE EFFECTS OF ATOMIC RADIATION (UNSCEAR) - Sources and effects of ionizing radiation, 1977 report to the general assembly, with annexes. New York: United Nations, 1977, 725 p.

[44] UNITED NATIONS SCIENTIFIC COMMITTEE ON THE EFFECTS OF ATOMIC RADIATION (UNSCEAR) - Sources, effects and risks of ionizing radiation, 1988 report to the general assembly, with annexes. New York: United Nations, 1988, 647 p.

[45] USSR STATE COMMITTEE ON THE UTILIZATION OF ATOMIC ENERGY The accident at the Chernobyl nuclear power plant and its consequences, information compiled fot the IAEA experts' meeting, Vienna, 25-29 Aug. 1986. Part II, annex 7 : Medical - biological problems. 